\title{
Prevalence and Determinants of Timely Initiation of Breastfeeding Among Mothers in Sodo Zuriya District, South Ethiopia: A Cross-sectional Study
}

\author{
Desta Mota Kuma $^{1}$, Abebe Sorsa Badacho ${ }^{2,}$, , Melese Meleku Kurucho ${ }^{3}$ \\ ${ }^{1}$ Siloam Health Sciences and Business College, Wolaita Sodo, Ethiopia \\ ${ }^{2}$ School of Public Health, College of Health Sciences and Medicine, Wolaita Sodo University, Sodo, Ethiopia \\ ${ }^{3}$ Department of Nursing, College of Health Sciences and Medicine, Wolaita Sodo University, Sodo, Ethiopia \\ Email address: \\ destamota@gmail.com (D. M. Kuma), sorsabebe@gmail.com (A. S. Badacho), melesemeleku@gmail.com (M. M. Kurucho) \\ ${ }^{*}$ Corresponding author
}

\section{To cite this article:}

Desta Mota Kuma, Abebe Sorsa Badacho, Melese Meleku Kurucho. Prevalence and Determinants of Timely Initiation of Breastfeeding Among Mothers in Sodo Zuriya District, South Ethiopia: A Cross-sectional Study. American Journal of Laboratory Medicine.

Vol. 5, No. 3, 2020, pp. 63-69. doi: 10.11648/j.ajlm.20200503.11

Received: June 6, 2020; Accepted: June 19, 2020; Published: July 28, 2020

\begin{abstract}
Timely initiation of breastfeeding tops the list of life-saving interventions for newborn health. Even though most mothers in Ethiopia breastfeed their babies, they do not always follow the recommendations of the "National Strategy for Infant and Young Child Feeding". A community-based cross-sectional study with internal comparisons was conducted between July and August 2019. A total of 444 mother-infant pairs were selected using simple random sampling. Descriptive statistics and bivariate and multivariable logistic regression were employed to identify predictors of timely initiation of breastfeeding. The prevalence of timely initiation of breastfeeding was 53.4\%. Institutional delivery (AOR=3.0; 95\% CI 1.47, 6.13), advice/counseling during antenatal visits $(\mathrm{AOR}=3.52 ; 95 \% \mathrm{CI} 1.81,6.83)$ and postnatal counseling/advice ( $\mathrm{AOR}=2.96 ; 95 \%$ CI $1.59,5.5)$ were found to be positively associated with timely initiation of breastfeeding $(\mathrm{p}<0.05)$. Conclusions and Recommendations: The prevalence of timely initiation of breastfeeding was low. Several characteristics affect timely initiation of breastfeeding. Educational programs in health institutions and at the community level are recommended to correct traditional inappropriate breastfeeding practices and beliefs and to provide training to health professionals to promote appropriate and timely counseling during the antenatal and postnatal periods.
\end{abstract}

Keywords: Timely Initiation, Breastfeed, Institutional Delivery, Within One Hour

\section{Background}

Timely initiation of breastfeeding is defined as putting the newborn to the breast within one hour of birth. Timely initiation of breastfeeding is not only easy and cost effective, it also tops the table of life-saving interventions for the health of the newborn $[1,2]$.

Optimal breastfeeding is one of the most effective preventive health measures against diarrheal diseases and child mortality. Breast milk protects infants from infectious and chronic diseases $[3,4]$. Twenty-two percent of neonatal deaths could be prevented if all infants were put to the breast within the first hour of birth [5].

The World Health Organization (WHO) has described breastfeeding as unequalled in terms of providing ideal food for the survival, healthy growth, and development of infants and young children. It is also an integral part of the reproductive process, with important implications for the health of mothers. The WHO and UNICEF have made it a global public health recommendation that breastfeeding should begin within one hour of birth. Feeding of colostrum should be promoted and pre-lacteal feeds discouraged. Colostrum is three times richer in vitamin A and ten times richer in beta-carotene than mature milk and, due to its high levels of vitamin A, antibodies, and other protective factors, colostrum is often considered the baby's first immunization 
[6].

The single most cost-effective intervention to reduce child mortality both in developed and developing countries is promotion of optimal breastfeeding practices. As noted above, breast milk provides the ultimate nutrition for a growing child via its many nutritional and immunological benefits, which prepares the growing child's immune response to infections. Despite all these advantages, the prevalence of breastfeeding is declining worldwide, and there is therefore a need to promote, protect, and support breastfeeding as a child survival strategy. More than 10 million children under the age of five die each year; $41 \%$ of these deaths occur in sub-Saharan Africa and another 34\% in South Asia. A major contributor to their deaths is poor breastfeeding practices [6].

Studies conducted in developing countries have shown that infants who are not breastfed are six- to ten-times more likely to die in the first month of life than infants who are breastfed. Diarrhea and pneumonia are more common and more severe in children who are artificially fed and are responsible for many of these deaths. It is estimated that sub-optimal breastfeeding, especially delayed initiation of breastfeeding within the first hour after birth and non-exclusive breastfeeding in the first six months of life, results in 1.4 million deaths and $10 \%$ of the disease burden in children younger than five years [7].

Even though feeding a baby with mother's milk is a well accepted and praised behavior in Ethiopian culture, the recommendations of the "National Strategy for IYCF", the guidelines established by the WHO and adopted by the Ethiopian FMOH for optimal breastfeeding, are not always followed. Many newborns are neither breast fed during their first hour of life with colostrum nor exclusively breast fed during their first six months [8].

There are several reasons for sub-optimal breastfeeding practices in Ethiopia including traditional and cultural beliefs, low educational levels, heavy workloads of mothers, poor sanitary conditions, the type of assistance given at delivery, duration of stay at home, ethnicity, poor maternal knowledge, age, parity, antenatal care service utilization, and place of delivery $[9,10]$.

The Ministry of Health in Ethiopia has tried to enhance optimal breastfeeding practices by developing training manuals and implementing guidelines on breastfeeding. These have been incorporated into primary healthcare in line with the health extension program, but still the practice is far from global [11].

Therefore, this study aimed to assess the prevalence and determinants of timely initiation of breastfeeding among mothers of infants. Although breastfeeding is one of the components of primary healthcare in Ethiopia, a wide range of harmful infant feeding practices have been documented even after implementation of infant and young child feeding recommendations [12]. Despite a few local studies conducted in different parts of the country, this is the first to identify factors associated with timely initiation of breastfeeding practices in the study area.

\section{Methods}

\subsection{Study Area and Period}

The study was conducted in Soddo Zuria district, South Ethiopia. The district has 31 rural kebeles (the smallest administrative unit in Ethiopia) with an estimated total population of 192,009 . In this region there were seven health centers and 35 health posts owned by the government, 1 health center owned by an NGO, and 16 private clinics. The study was conducted from July 2019 to August 2019.

\subsection{Study Design}

A community-based cross-sectional study with internal comparisons.

\subsection{Sample Size Determination}

Sample size was calculated using Open-EPI sample size calculator software using $95 \%$ confidence intervals (CI), a proportion of timely initiation of breastfeeding of $52 \%$ [13], total under one year child population 6,259 , and a margin of error of $5 \%$. The sample size with Fleiss continuity correction was 362 , and with a $10 \%$ non-respondent rate, the total sample size was calculated as 398 . The sample size for factors affecting timely initiation of breastfeeding was calculated using EPI-Info sample size calculator software version 3.3.2 with assumptions based on the recent study conducted at Goba Woreda, South East Ethiopia: antenatal counselling $56.8 \%$ unexposed (OR 2.0), postnatal counselling $60.2 \%$ unexposed (OR 2.7), and institutional delivery unexposed $63.9 \%$ (OR 1.9) [14]. With a nonresponse rate of $10 \%$, the largest calculated total sample size was 444.

\subsection{Sampling Techniques}

444 respondents were selected using simple random sampling.

\subsection{Data Collection}

The definitions and recommendations of the WHO and the National Strategy for IYCF were used for the assessment of timely initiation of breastfeeding practices. The questionnaire was in three parts: the first collected sociodemographic characteristics of mothers and children; the second maternal health-related practices; and the third the feeding practices of the child. The questionnaire was designed to assess the experience of timely initiation of breastfeeding and the factors associated with the timely initiation of breastfeeding practices. The mothers were requested to provide information regarding the time at which their index infant was put to the breast after delivery. Six data collectors and two supervisors with more field experience were trained. Interviews were performed faceto-face using the pre-tested structured questionnaire adapted from the literature [7, 13]. Data on timely initiation of breastfeeding practices were collected from mothers with 
children less than one year of age.

\subsection{Data Quality Control}

Questionnaire was prepared in English and translated into the local language (Amharic) and back to English by two different experts for consistency. Training was given to data collectors and supervisors. The questionnaire was pretested.

\subsection{Data Analysis}

Data obtained from the field survey was checked for completeness and inconsistencies and then coded, entered, cleaned by Epi-Info, and analyzed using SPSS for Windows version 16.0 (IBM Corps., Chicago, IL). Data were summarized with tables and figures. Descriptive statistics were computed to determine the prevalence of timely initiation of breastfeeding. Proportions were compared by timely initiation of breastfeeding using chi-squared tests. First, bivariate logistic regression was performed. Variables that showed significant associations with timely initiation of breastfeeding in the bivariate models were entered in a multivariable logistic regression model. To identify independent predictors of timely initiation of breastfeeding, a multivariable logistic regression model with timely initiation of breastfeeding as the dependent variable was constructed. The significance level was measured using crude and adjusted odds ratios (ORs) with $95 \%$ CIs, and $\mathrm{p}<0.05$ was considered significant.

\subsection{Operational Definitions}

Timely initiation of breastfeeding: putting the neonate on the mother's breast to suckle within one hour (including one hour).

Antenatal counseling: advice/counseling on breastfeeding provided by healthcare professionals to pregnant mothers during antenatal care (ANC) visits.

Postnatal counseling: advice/counseling on breastfeeding provided by healthcare professionals to mothers immediately giving birth during the antenatal care visit.

Parity: the number of live births.

Ever breastfeeding: breastfeeding at any point in time since birth.

\subsection{Ethical Approval}

Ethical approval was obtained from the ethical clearance board of Wolaita Sodo University (reference number RPGC/445/2019). The participants were informed about the purpose of the study and oral consent was obtained from each study participant prior to conducting the interview.

\section{Results}

\subsection{Socio-demographic Characteristics}

A total of 444 mothers with children aged 12 months and less were interviewed (response rate 100\%). The mean age of mothers was $27.6( \pm 5.1)$. Over a third $(154 ; 34.7 \%)$ of mothers were aged between 25 and 29 years. Three hundred and one $(67.8 \%)$ were Protestant, and the dominant ethnic group was Wolaita $(435 ; 98.0 \%)$.

With respect to educational status, 317 (71.4\%) had attended formal school, of whom 124 (27.9\%) had accomplished secondary school and higher (grade 9 and above). The majority of mothers were married (435; 98.0\%) and were housewives by occupation $(372 ; 83.8 \%)$. Over half $(256 ; 57.9 \%)$ earned an average monthly income of $\leq 501$ Birr. About 85.1\% (378) husbands attended formal education (Table 1).

Table 1. Socio-demographic and economic characteristics of breastfeeding mothers in Sodo Zuria District, Wolaita Zone, South Ethiopia, 2019.

\begin{tabular}{|c|c|c|c|}
\hline Variable & Category & Frequency & $\%$ \\
\hline \multirow[t]{5}{*}{ Age (years) } & $15-19$ & 9 & 2.0 \\
\hline & $20-24$ & 83 & 18.7 \\
\hline & $25-29$ & 154 & 34.7 \\
\hline & $30-34$ & 132 & 29.7 \\
\hline & $35+$ & 66 & 14.9 \\
\hline \multirow[t]{3}{*}{ Ethnicity } & Wolaita & 435 & 98.0 \\
\hline & Gamo & 4 & .9 \\
\hline & Other* & 5 & 1.1 \\
\hline \multirow[t]{3}{*}{ Marital status } & Married & 435 & 98.0 \\
\hline & Never married & 4 & .9 \\
\hline & Other** & 5 & 1.1 \\
\hline \multirow[t]{4}{*}{ Religion } & Protestant & 301 & 67.8 \\
\hline & Orthodox & 125 & 28.2 \\
\hline & Muslim & 6 & 1.4 \\
\hline & Catholic & 12 & 2.7 \\
\hline \multirow[t]{4}{*}{$\begin{array}{l}\text { Educational } \\
\text { status of mothers }\end{array}$} & No formal education & 119 & 26.8 \\
\hline & Read and write & 8 & 1.8 \\
\hline & Primary (Grade 1-8) & 193 & 43.5 \\
\hline & $\begin{array}{l}\text { Secondary and above } \\
(9+)\end{array}$ & 124 & 27.9 \\
\hline \multirow[t]{2}{*}{ Access to media } & Yes & 187 & 42.1 \\
\hline & No & 257 & 57.9 \\
\hline \multirow[t]{5}{*}{ Occupation } & House wife & 372 & 83.8 \\
\hline & Gov. employed & 8 & 1.8 \\
\hline & Business woman & 52 & 11.7 \\
\hline & Farmer & 8 & 1.8 \\
\hline & Other*** & 4 & .9 \\
\hline \multirow[t]{4}{*}{ Monthly income } & $\leq 500$ & 257 & 57.9 \\
\hline & $5001-1000$ & 123 & 27.7 \\
\hline & $>1000$ & 38 & 8.6 \\
\hline & Do not know & 26 & 5.9 \\
\hline \multirow[t]{2}{*}{ Sex of infant } & Male & 227 & 51.1 \\
\hline & Female & 217 & 48.9 \\
\hline \multirow[t]{2}{*}{ Age of infant } & $<6$ months & 203 & 45.7 \\
\hline & $>6$ months & 241 & 54.3 \\
\hline
\end{tabular}

*Amahra, Gurage, Oromo **divorced, widowed, separated *** Daily laborer, private organization employee.

\subsection{Obstetrics and Health Service-related Variables}

For the current child, $321(72.3 \%)$ had received ANC services at least once, of whom 246 (76.6\%) received advice/counseling on breastfeeding during their ANC visit. Three-quarters $(74.5 \%)$ of mothers gave birth to their current child at home with the assistance of traditional birth attendants and relatives, and about a quarter $(113 ; 25.5 \%)$ 
delivered in health institutions (Table 1).

\subsection{Pattern of Breastfeeding Practices}

\subsubsection{Ever Breastfeeding Rate}

All of the respondents (100\%) practiced ever breastfeeding for their current child.

\subsubsection{Prevalence of Timely Initiation of Breastfeeding}

All the study subjects were asked whether they had ever breastfed, and for those who had ever breastfed they were also asked the time of initiation of breastfeeding of their index child. Over half $(53.4 \%)$ of mothers initiated breastfeeding within one hour (including one hour) after delivery, and $40.3 \%$ of them initiated breastfeeding with one hour to one day. Only $28 \quad(6.3 \%)$ mothers initiated breastfeeding at 1 to 3 days.

\subsubsection{Determinants of Breastfeeding Initiation}

Timely initiation of breastfeeding was assessed for its association with socio-demographic, obstetric, and health service-related variables. Attending formal education was significantly associated with timely initiation of breastfeeding $(\mathrm{p}<0.01)$; mothers who had formal education were 1.76-times more likely to initiate breastfeeding with in the first hour compared to those who had no formal education $(\mathrm{COR}=1.76$; CIs 1.16, 2.67). Likewise, mothers who had access to media were 2.9-times more likely to initiate breastfeeding $(\mathrm{COR}=2.92$; CIs 1.97, 4.34; $\mathrm{p}<0.001)$. Among obstetric and health-related factors, advice during ANC visit was statistically associated with timely initiation of breastfeeding, which were $72.8 \%$ and $29.3 \%$, respectively $(\mathrm{p}<0.001)$. Mothers who had received advice/counseling during ANC follow-up were 6.4-times more likely to initiate breastfeeding within one hour than those who had not $(\mathrm{COR}=6.43$; CIs $3.63,11.39)$. Place of delivery and postnatal counseling were associated with timely initiation of breastfeeding $(p<0.01)$. Mothers who gave birth in health institutions were 7.61-times more likely to initiate breastfeeding in a timely manner than those who delivered at home $(\mathrm{COR}=7.61$; CIs 4.34, 13.32). Similarly, mothers who were counseled during the postnatal period were 8.6-times more likely to initiate breastfeeding compared to those who had not been counseled ( $\mathrm{COR}=8.61$; CIs 5.46, 13.57). There was also a difference with regard to delivery assistance; mothers assisted by health professionals were more likely to initiate timely breastfeeding than those assisted by non-health professionals (TBA, relatives; $\mathrm{COR}=7.02$; CIs 4.05, 12.16) (Table 2).

Table 2. Distribution of obstetrics and health service-related variables of mothers in Soddo Zuria District, South Ethiopia, 2019 ( $n=444)$.

\begin{tabular}{|c|c|c|c|}
\hline Variables & Category & Frequency & $\%$ \\
\hline \multirow{3}{*}{ Birth interval (year) } & 1 & 12 & 3.3 \\
\hline & $2-3$ & 200 & 56.4 \\
\hline & $4 \&$ above & 142 & 40.1 \\
\hline \multirow[t]{2}{*}{ History of ANC } & Yes & 321 & 72.3 \\
\hline & No & 123 & 27.7 \\
\hline \multirow[t]{3}{*}{ Number of ANC visits } & 1 & 123 & 38.3 \\
\hline & $2-3$ & 163 & 50.8 \\
\hline & $>=4$ & 35 & 10.9 \\
\hline \multirow{2}{*}{$\begin{array}{l}\text { Health information provided } \\
\text { on BF during ANC visit }\end{array}$} & Yes & 246 & 76.6 \\
\hline & No & 75 & 23.4 \\
\hline \multirow[t]{3}{*}{ Place of delivery } & Home & 331 & 74.5 \\
\hline & Hospital & 30 & 6.8 \\
\hline & Health center & 83 & 18.7 \\
\hline \multirow[t]{2}{*}{ Mode of delivery } & Vaginal & 436 & 98.2 \\
\hline & $\mathrm{SC}$ & 8 & 1.8 \\
\hline \multirow[t]{3}{*}{ Birth attendant } & TBA & 75 & 16.9 \\
\hline & Health professional & 113 & 25.5 \\
\hline & Relative & 256 & 57.7 \\
\hline \multirow[t]{2}{*}{ Post natal follow up } & Yes & 168 & 37.8 \\
\hline & No & 276 & 62.2 \\
\hline
\end{tabular}

Table 3. Socio-demographic obstetric and health service-related factors versus timely initiation of breast feeding in Sodo Zuriya District, South Ethiopia, 2019 $(n=444)$.

\begin{tabular}{|c|c|c|c|c|c|}
\hline \multirow{2}{*}{ Variable } & \multirow{2}{*}{ Category } & \multicolumn{4}{|c|}{ Timely initiation of breast feeding } \\
\hline & & Yes, n (\%) & No $(\%)$ & COR $(95 \% \mathrm{CI})$ & AOR (95\% CI) \\
\hline \multirow[t]{5}{*}{ Age (in years) } & $15-19$ & $5(55.6)$ & $4(44.4)$ & $1.04[.25,4.22$ & $0.99[0.24,4.11]$ \\
\hline & $20-24$ & $48(57.8)$ & $35(42.2)$ & $1.14[0.59,2.19$ & $1.08[0.56,2.1]$ \\
\hline & $25-29$ & $85(55.2)$ & $69(44.8)$ & $1.02[0.57,1.83]$ & $0.92[0.51,1.672]$ \\
\hline & $30-34$ & $63(47.7)$ & $69(52.3)$ & $0.76[0.42,1.37]$ & $0.69[0.381 .27]$ \\
\hline & $35+$ & $36(55.5)$ & $30(45.5)$ & 1.0 & 1.0 \\
\hline \multirow[t]{2}{*}{ Marital status } & Married & $231(53.1)$ & $204(46.9)$ & $0.56[0.14,2.29]$ & $0.77[0.18,3.28]$ \\
\hline & Not married & $6(66.7)$ & $3(33.3)$ & 1.0 & 1.0 \\
\hline \multirow[t]{2}{*}{ Literacy } & Educated & $182(57.4)$ & $135(42.6)$ & $1.76[1.16,2.67]^{* *}$ & $1.07[0.54,2.130]$ \\
\hline & Not educated & $55(43.3)$ & $72(56.7)$ & 1.0 & 1.0 \\
\hline \multirow[t]{2}{*}{ Occupation } & Not working mother & $192(51.3)$ & $180(48.7)$ & $0.64[0.38,1.07]$ & $0.58[0.27,1.23]$ \\
\hline & Working mother & $45(62.5)$ & $27(37.5)$ & 1.0 & 1.0 \\
\hline \multirow[t]{2}{*}{ Access to media } & Yes & $128(68.4)$ & $59(31.6)$ & $2.92[1.97,4.34] * * *$ & $1.70[0.97,2.97]$ \\
\hline & No & $109(42.3)$ & $147(57.7)$ & 1.0 & 1.0 \\
\hline \multirow[t]{3}{*}{ Monthly income } & $\leq 500$ & $139(49.1)$ & $144(50.9)$ & $0.63[0.31,1.25$ & $1.35[0.47,3.86]$ \\
\hline & $5001-1000$ & $75(61)$ & $48(39.0)$ & $1.01[0.48,2.14]$ & $1.05[0.49,2.25]$ \\
\hline & $>1000$ & $23(60.5)$ & $15(39.5)$ & 1.0 & 1.0 \\
\hline \multirow[t]{2}{*}{ Sex of infant } & Male & $121(53.3)$ & $106(45.7)$ & $0.99[0.68,1.44]$ & $1.01[0.694,1.48]$ \\
\hline & Female & $116(51.1)$ & $101(48.9)$ & 1.0 & 1.0 \\
\hline \multirow[t]{2}{*}{ Birth interval } & 1 year & $5(41.7)$ & $7(58.3)$ & $0.58[0.17,1.93]$ & $0.55[0.16,1.87]$ \\
\hline & $2-3$ years & $97(48.5)$ & $103(51.5)$ & $0.77[0.50,1.19]$ & $0.77[0.49,1.22]$ \\
\hline
\end{tabular}




\begin{tabular}{|c|c|c|c|c|c|}
\hline \multirow{2}{*}{ Variable } & \multirow{2}{*}{ Category } & \multicolumn{4}{|c|}{ Timely initiation of breast feeding } \\
\hline & & Yes, n (\%) & No (\%) & COR $(95 \%$ CI) & AOR (95\% CI) \\
\hline \multirow{4}{*}{ No. of ANC visits } & $4 \&$ above & $78(54.9)$ & $64(45.1)$ & 1.0 & 1.0 \\
\hline & 1 & $64(52.9)$ & $57(47.1)$ & $0.59[0.25,1.37]$ & $1.22[0.44,3.40]$ \\
\hline & $2-3$ & $100(67.1)$ & $49(32.9)$ & $1.07[0.46,2.48]$ & $1.88[0.689,5.17]$ \\
\hline & $>=4$ & $19(65.5)$ & $10(34.5)$ & 1.0 & 1.0 \\
\hline \multirow[t]{2}{*}{ ANC counseling on BF } & Yes & $179(72.8)$ & $67(27.2)$ & $6.43[3.63,11.39]^{* * *}$ & $3.52[1.81,6.83]^{* * *}$ \\
\hline & No & $22(29.3)$ & $53(70.7)$ & 1.0 & 1.0 \\
\hline \multirow[t]{2}{*}{ Place of delivery } & Home & $141(42.6)$ & $190(57.4)$ & 1.0 & 1.0 \\
\hline & Institutional & $96(85.0)$ & $17(15.0)$ & $7.61[4.34,13.32]^{* *}$ & $3.01[1.47,6.13]^{* *}$ \\
\hline \multirow[t]{2}{*}{ Mode of delivery } & Vaginal & $92(87.6)$ & $13(12.4)$ & $1.14(0.28,4.64)$ & $1.24[0.16,9.58]$ \\
\hline & $\mathrm{C} / \mathrm{s}$ & $4(50.0)$ & $4(50.0)$ & 1.0 & 1.0 \\
\hline \multirow[t]{2}{*}{ Birth attendant } & Non H. professional & $142(43.0)$ & $189(57.0)$ & 1.0 & 1.0 \\
\hline & Health professional & $95(84.0)$ & $18(16.0)$ & $7.02[4.05,12.16]^{* * *}$ & $0.20[0.01,3.02]$ \\
\hline \multirow[t]{2}{*}{ PNC counseling on BF } & Yes & $147(81.7)$ & $33(18.3)$ & $8.61[5.46,13.57]^{* * *}$ & $2.96[1.59,5.5]^{* *}$ \\
\hline & No & $90(34.0)$ & $174(66.0)$ & 1.0 & 1.0 \\
\hline
\end{tabular}

${ }^{* *} \mathrm{p}<0.01 * * * \mathrm{p}<0.001 \mathrm{COR}$ - crude odds ratio AOR - Adjusted odds ratio CI = confidence interval

In multivariate analysis, after adjusting for all possible confounding variables, institutional delivery ( $\mathrm{AOR}=3.01$; CIs $1.47,6.13)$, mothers advised on breastfeeding in the antenatal period $(\mathrm{AOR}=3.5$; $\mathrm{CI} 1.81,6.83)$, and mothers who were counseled on breastfeeding in the postnatal period $(\mathrm{AOR}=2.96$; CIs $1.59,5.5)$ were positively associated with timely initiation of breastfeeding (Table 3).

\section{Discussion}

This study revealed that all respondents practiced breastfeeding for their current child, similar to a study from Ghana [15], Cameroon, and the Goba district, South East Ethiopia. The national ever breastfeeding rate is high at $98 \%$ [13], higher than the ever breastfeeding rate in Nigeria (82\%) [16] and the US (73.8\%) [17]. This high rate of breastfeeding is likely to be due to due to breastfeeding being normal in this society.

Global and national infant and young child feeding guidelines recommend that all newborns should start breastfeeding immediately (with in the first hour after delivery) and that the feeding of colostrum should be promoted. Here, 164 (36.9\%) mothers squeezed and discarded colostrum, in part due to cultural beliefs that colostrum is considered dirty and causes abdominal cramping in infants. In this study, the prevalence of pre-lacteal feeding was $18.7 \%$, lower than the national pre-lacteal feeding prevalence of $29 \%$ [13]. The reasons cited for giving prelacteal feeds were colostrum causing abdominal cramps, breast milk insufficiencies, and maternal illness.

The prevalence of timely initiation of breastfeeding was $53.4 \%$, higher than similar studies in Bangladesh (24.2\%), Cameroon (31.9\%), Burkina Faso (33.3\%), Jordan (39.7\%), Chad (43.3\%), Colombia (48.9\%), Nepal (35.4\%), and India (36\%). Only Eritrea (77.9\%) and Namibia (80.9\%) [18-20] some regions in Ethiopia (SNNPR, Dredawa; 66\% and $66.5 \%$, respectively) reported higher rates [13]. Findings from Morocco (52\%), the Goba district in South East Ethiopia $(52.4 \%)$, and national statistics $(52 \%)$ were similar $[13,14]$.

Overall, mothers who gave birth in health institutions were better at practicing timely initiation. Related to this, the proportion of children who breastfed within an hour varied significantly according to the type of assistance given to mothers during delivery: timely feeding was higher in those assisted by health professionals than non-health professionals. This finding is consistent with a previous study conducted in the Goba district, Ethiopia, but contrasts with data from the Ethiopian Demographic Health Survey, which indicated that place of delivery and type of birth assistance did not have a significant impact on the timely initiation of breastfeeding [13, 14].

Place of delivery and receiving advice/counseling on breastfeeding practice during antenatal and postnatal visits were independent predictors of timely initiation of breastfeeding. Mothers who gave birth at health institution were three-times more likely to initiate breastfeeding compared to those delivering at home. Mothers receiving antenatal counseling on breastfeeding were more likely to initiate breastfeeding in an hour compared to mothers who did not. Likewise, mothers who received advice/counseling on breastfeeding in the post-natal period were more likely to initiate breastfeeding in an hour. This might be related to the fact that mothers are exposed to breastfeeding information during postnatal visits, which is the most appropriate time for delivering key infant and young child feeding messages [1, 4].

Ethiopia has developed National Infant and Young Child Feeding Guidelines with the objective of achieving optimal breastfeeding. For their implementation, many health extension workers were trained to provide community-level breastfeeding promotion, and in-service training has been given to promote breast-feeding at the health facility level. Despite these interventions, the basic and easiest indicator of optimal breastfeeding (i.e., timely initiation of breastfeeding) remains at near average levels and falls short of the HSDP IV target of $92 \%$. Furthermore, timely initiation is predicted by nutrition behavior change communication delivered during the antenatal and postnatal periods, implying a need to strengthen the delivery of key messages for optimal breastfeeding during antenatal and postnatal contacts both at the health institution and at health post levels 


\section{Limitations of the Study}

A mother may have difficulty in remembering exactly when she initiated breastfeeding; as a result, timely initiation of breastfeeding is subject to potential recall bias. The study design (cross-sectional) will not explain any causal relationships between any of the factors assessed.

\section{Conclusion}

This study revealed that the timely initiation of breastfeeding was low, and many mothers introduce prelacteal foods due to some traditional practices and beliefs. Attending formal education, access to media counseling/advice on breastfeeding during ANC visits, postnatal counseling, place of delivery, and birth attendants were significantly associated with timely initiation of breastfeeding in bivariate analyses. Of these, place of delivery and counseling/advice on breastfeeding during ANC and postnatal visits were predictors of timely initiation of breastfeeding.

\section{Recommendations}

We propose the following recommendations:

1. Educational programs in health institutions and at the community level should be targeted to correct traditional inappropriate breastfeeding practices and beliefs.

2. Coordinating, strengthening, and sustaining existing strategies and developing approaches for further improvement of optimal breastfeeding practice are important.

3. Training must be given to health professionals to promote appropriate and timely counseling during ANC and postnatal visits.

4. Further studies should examine these associated factors at the individual, group, and societal levels.

\section{Declaration}

\section{Ethics and Consent to Participate}

Ethical approval was obtained from the ethical clearance board of Wolaita Sodo University with reference number RPGC/445/2019, according to the standardized principles and procedures of national and WHO guidelines.

The participants were informed about the purpose of the study and oral consent was obtained from each study participant.

\section{Consent for Publication}

Not applicable

\section{Availability of Data and Material}

Dataset generated and analyzed during the study is available.

\section{Competing Interests}

The authors declare that they have no competing interests.

\section{Funding}

Nil

\section{Authors' Contributions}

DMK, ASB and MMK: conception and design of the study and data analysis. DMK; ASB analyzed the data and interpreted the findings, conducted and supervised data collection and management. All the authors read and approved the final manuscript.

\section{Acknowledgements}

The author's sincere gratitude goes to the data collectors, supervisors and study participants. We also thank the Nextgenediting Global Initiative (www.nextgenediting.com) for editorial help.

\section{Author Information}

ASB: Assistant professor of public health, Wolaita Sodo University, College of Health Sciences and Medicine, School of Public Health, P. O. Box 138;

DMK: Lecturer, Siloam Health science and Business College;

MMK: Lecturer, Wolaita Sodo University, College of Health Sciences and Medicine, Department of Nursing, P. O. Box 138,

\section{References}

[1] A, K., H. R, and M. N, Breast-feeding patterns and factors determining exclusive breast-feeding. Singapore Med J 2008. 49 (12): p. 1002-006.

[2] FMOH and UNICEF, join forces to promote safe breastfeeding. 6 august 2004 .

[3] KM, J. and N. AM, Breastfeeding, the Immune Response, and Long term Health JAO 2006. 106 (4): p. 203-207.

[4] WHO Geneva, G., Switzerland: Global Strategy for Infant and Young Child Feeding. 2003, A joint WHO/UNICEF statement: Geneva WHO.

[5] Ministry of finance and economic development population department and an annotated bibliography of population and reproductive health researches in Ethiopia. December, 2008 p. 24-28.

[6] organization, W. h., fifty-fifth world health assembly, provisional agenda item 13.10 Infant and young child nutrition Global strategy on infant and young child feeding 16 April 2002, Report by the Secretariat.

[7] WHO, Infant and young child feeding: model chapter for textbooks for medical students and allied health professionals 2009. 
[8] Agency, C. S., Demographic and health survey 2005, Ethiopia ORC Macro Calverton September 2006: Maryland, USA.

[9] D, D. P., Breastfeeding: Mothers and health practitioners in the context of private medical care in Gauteng. Journal of Interdisciplinary Health Sciences, 2009. 14 (1).

[10] KM, E., Z. C, and Q. MA, Delayed breastfeeding initiation increases risk of neonatal mortality. Pediatrics 2006. 117: p. 380-86.

[11] (FMOH), F. M. o. H., National strategy for Infant and Young Child Feeding (IYCF). 2004, Family Health Department Ethiopia.

[12] Tamiru and etal, sub optimal breast feeding of infants during the first six months and associated factors in rural communities of Jima Arjo woreda south west Ethiopia. PH, 2012. 12: p. 363.

[13] Agency, C. S., Demographic and health survey 2011 March 2012, Ethiopia ICF International Calverton Maryland USA.

[14] Setegn and et al, determinants of timely initiation of breast feeding among mothers in Goba woreda South Ethiopia Ph, April 2011. 11: p. 2017.
[15] B, S., Knowledge, Attitude and Practice of Breast Feeding - A Case Study. European Journal of Scientific Research, 2010. 40 (3): p. 404-422.

[16] L, S., factors influencing breastfeeding practices Edo state, Nigeria. African journal of food, agriculture, nutrition and development, 2006. 6 (2).

[17] M, M., et al., Breastfeeding in the United States: Findings from the National Health and Nutri tion Examination Surveys, 1999-2006, NCHS Data Brief, 2008. 5

[18] AG, M., K. MT, and A. N, Infant and Young Child Feeding Update 2006, ORC Macro Calverton, Maryland, USA.

[19] P, S., K. T, and U. S, Determinants of infant and young child feeding practices in Nepal: secondary data analysis of Demographic and Health Survey 2006. Food Nutr Bull 2010. 31 (2): p. 334-51.

[20] Ekambram and et al, knowledge attitude and practice of breast feeding among postnatal mothers. Curr pediatr Res, 2010. 14 (2): p. 119-124. 